DOI 10.17816/transsyst201844129-137

\author{
(C) Y. Yang, W. Zhang, L. Tong, Q. Peng, H. Luo, J. Suo \\ CRRC Zhuzhou Locomotive CO., LTD \\ (Zhuzhou, China)
}

\title{
ANALYSIS AND SOLUTION OF EDDY CURRENT INDUCED IN RAIL FOR MEDIUM AND LOW SPEED MAGLEV TRANSPORTATION SYSTEM
}

Background: For medium and low speed maglev transportation system, the eddy current will be induced in rail, which is made of solid steel, while the train is running. The levitation force of electromagnets will be weakened by the magnetic field generated by eddy current in the rail, especially at the position of the forefront electromagnets. With the increase of train running speed, the eddy current effect will also increase, which will reach $30 \%$ at $100 \mathrm{~km} / \mathrm{h}$, and which will directly affect the levitation stability of the train during high-speed running. Put it another way, it will limit the further improvement of the running speed of the medium and low speed maglev train.

Aim: In order to solve the above problem, and compensate the levitation force reduced by the eddy current effect.

Methods: The FEA method is used to obtain the magnetic field distribution and levitation force changing with the train speed. And taking the middle and low speed maglev trains and rails of Changsha Maglev Express as the research object, we have adopted two solutions, and the prototypes of airsprings and levitation magnets are manufactured and tested in the train.

Results: The test result show that the currents of the windings at the front end of the two forefront electromagnets are reduced obviously.

Conclusion: In this paper, the medium and low speed maglev train and rail used by Changsha Maglev Express are studied, the eddy current effect is analyzed, and two solutions are proposed. The results show that the solution methods can alleviate the eddy current effects to some extent.

Keywords: maglev transportation system, maglev train, eddy current, electromagnet

\section{INTRODUCTION}

Compared with traditional urban rail vehicles, medium and low speed maglev trains use electromagnetic force for support and guidance, and they are equipped with linear motors for drive. There is no mechanical contact between the vehicle and the rail, which has high running speed, low noise and strong climbing ability. With its significant advantages such as comfort and maintenance, it is one of the main directions for the development of urban rail vehicles in the future.

To reduce the rail manufacturing cost, the rail of the medium and low speed maglev transportation system generally adopts a solid structure. When the train is 
running, eddy currents are generated in the rail. The magnetic field generated by the rail eddy current will weaken the electromagnets' levitation force, especially the forefront electromagnet. Because it establishes the magnetic field first in the rail, the magnetic flux at that position changes the most, and the influence of the eddy current effect is also most significant. The test was conducted on the Changsha Maglev Express: the current of the forefront electromagnet was more than $30 \%$ higher than the average value of other electromagnet currents, resulting in serious heating of the forefront electromagnet.

As early as 2004 [1] analyzed and calculated the eddy current effect of maglev transportation systems and gave relationships between levitation forces, eddy currents, air gap magnetic density and velocities. However, literature 1 only gave analysis results and did not propose specific solutions.

In the dissertation of Chinese scientists Zheng Lili, Li Jie, Li Jinhui [2] used the analytical method and the finite element method to analyze the influence of rail eddy currents on the levitation forces of medium and low speed maglev trains and proposed to put the permanent magnets at the front of electromagnets to compensate for the loss of levitation forces. And a pilot study was conducted on the CMS-04 medium and low speed maglev train on the Tangshan test line in China.

However, permanent magnets have permanent magnetism. Therefore, levitation magnets using permanent magnets are prone to adsorb iron foreign materials. If they get stuck between the electromagnets and rail, it will seriously affect the safety of the train. Thence, no permanent magnet is used in the current commercial maglev trains.

To solve the above problems, taking the middle and low speed maglev trains and rails of Changsha Maglev Express as the research object, we have adopted two solutions: the first solution is to reduce the area of the air springs installed above the forefront electromagnets. Thus under the same air pressure, the load of this position can be reduced so that reducing the current of the levitation electromagnets in this position; the second solution is to increase the number of forefront electromagnet's windings and the length of the magnetic pole, thus its levitation capacity will be enhanced, and under the same load conditions, the current will drop.

This article will introduce the analysis and calculation of the above two solutions and experiments for them.

\section{RAIL EDDY CURRENT EFFECT ANALYSIS}

Changsha Maglev Express started being in commercial operation on May 6, 2016. At present, there are six trains operating on the line. By the end of June 2018, it has been in safe operation for 1.7 million kilometers and carried nearly 6 million passengers. It is called as version 1.0 commercial maglev train in China. 
As shown in Fig. 1, the medium and low speed maglev train of Changsha Maglev Express consists of three carriages. The train has a total length of $48 \mathrm{~m}$, a width of $2.8 \mathrm{~m}$, a maximum operating speed of $100 \mathrm{~km} / \mathrm{h}$, and a maximum carrying capacity of 363 passengers.

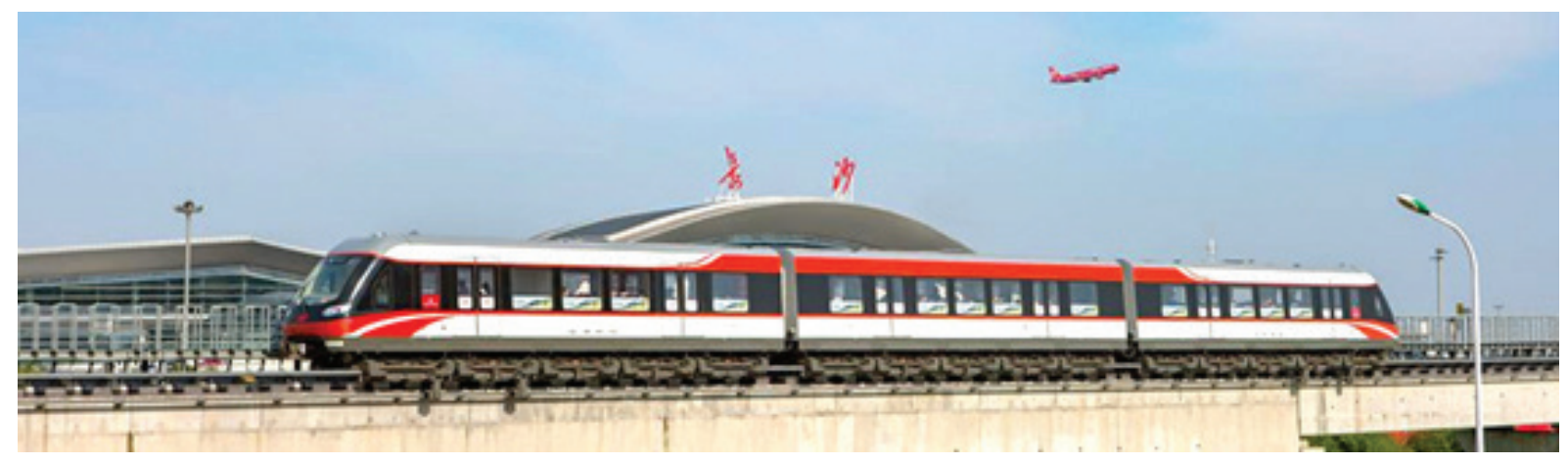

Fig. 1. Medium and low speed maglev train of Changsha Maglev Express

Fig. 2 shows the data of forefront electromagnet current on version 1.0 maglev train, while the average current of other electromagnets is $28.21 \mathrm{~A}$. As the speed increases, the forefront electromagnet current also increases, which is 1.54 times larger than in the stationary state and is 1.37 times larger than the average current of other electromagnets. The eddy current effect at the forefront electromagnet of the train leading to the decrease of the levitation force. To compensate the loss of levitation force, closed-loop control system will increase the current output automatically.

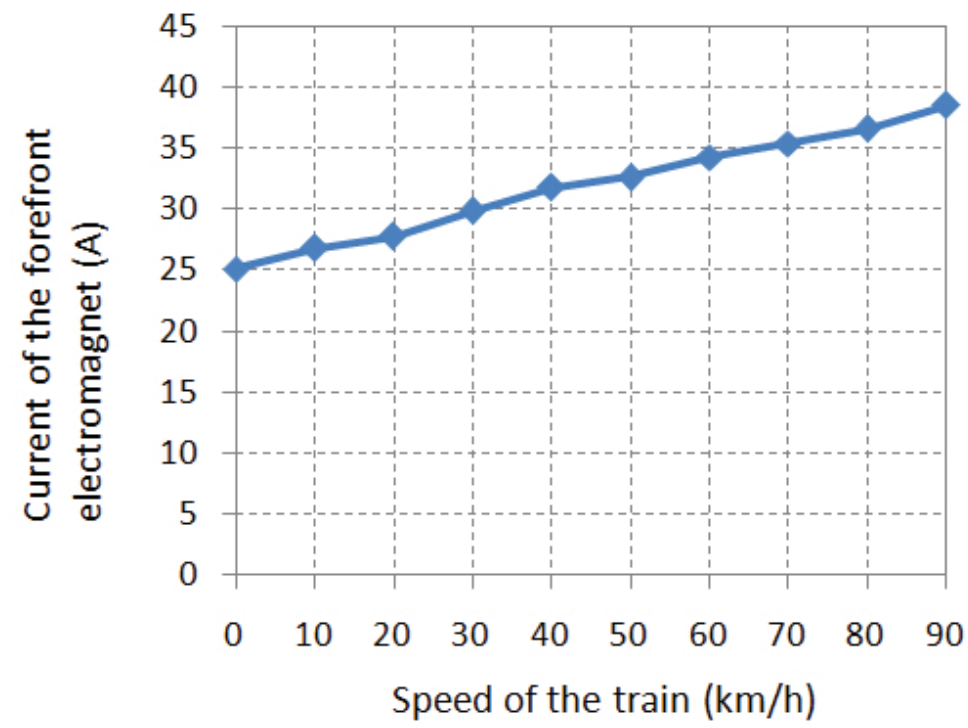

Fig. 2. Data of electromagnet current at different speeds of maglev train 
To fulfill the need of transportation between the center of city and the satellite cities, CRRC ZELC has designed and manufactured version 2.0 commercial maglev train with the speed grade of $160 \mathrm{~km} / \mathrm{h}$ based on version 1.0 commercial maglev train. As the increase of the speed, the eddy current effect at the end of the train increases heavily.

Because the version 2.0 commercial maglev train is still under commissioning, we only get the analysis results of the drop of levitation force when the speed of the train reaches $160 \mathrm{~km} / \mathrm{h}$.

The structure of rail and levitation electromagnet in Changsha is shown in Fig. 3. They both made of steel Q235, with the conductivity of $5 \times 10^{6} \mathrm{~S} / \mathrm{m}$, saturated magnetic density of $1.4 \mathrm{~T}$ and the density of $7850 \mathrm{~kg} / \mathrm{m}^{3}$. The B-H curve of steel Q235 is shown in Fig. 4.

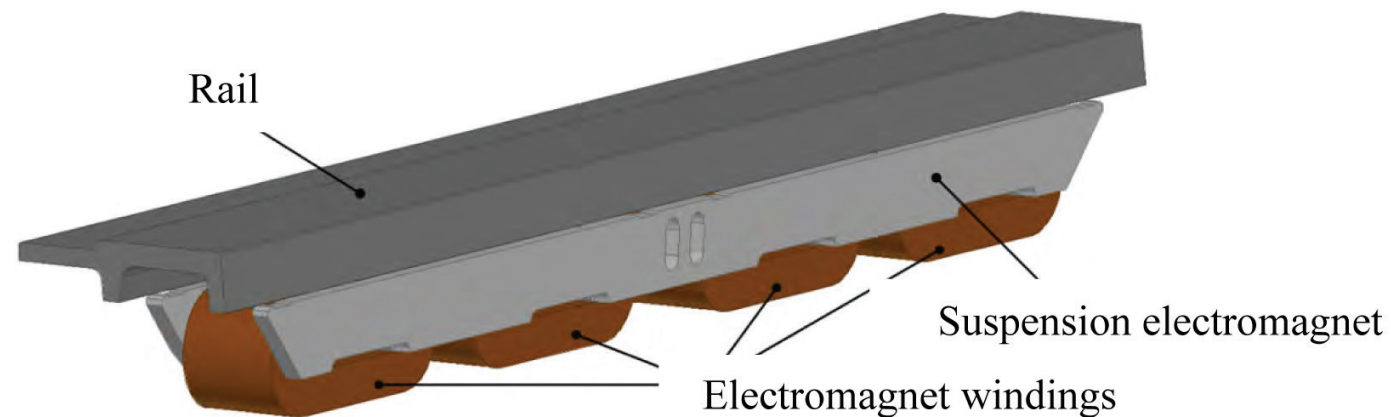

Fig. 3. Structure of rail and levitation electromagnet

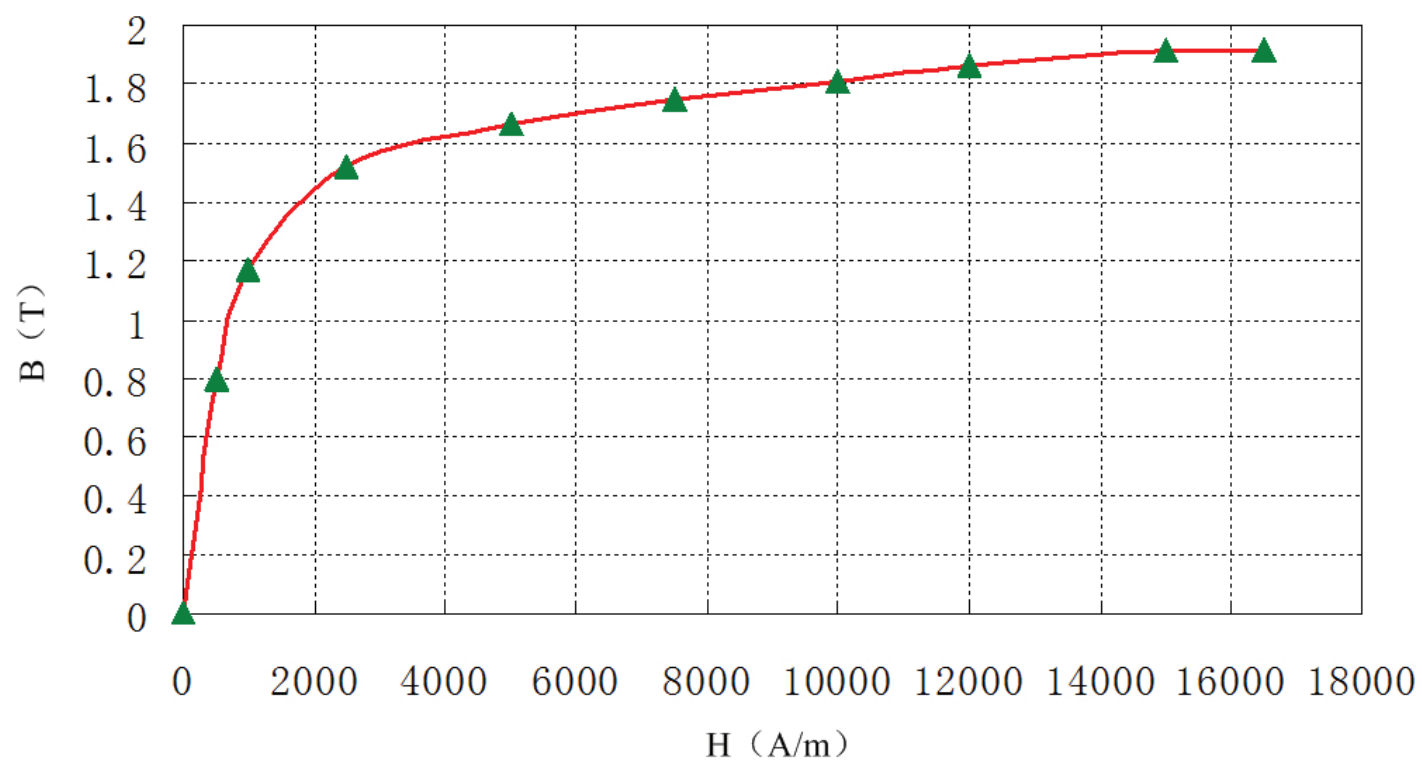

Fig. 4. B-H curve of Q235 
Combining the above structure and material properties, we use the threedimensional electromagnetic field finite element analysis to shows that the levitation force of the forefront electromagnet drops by nearly $40 \%$ at a speed of $160 \mathrm{~km} / \mathrm{h}$, as shown in Fig. 5.

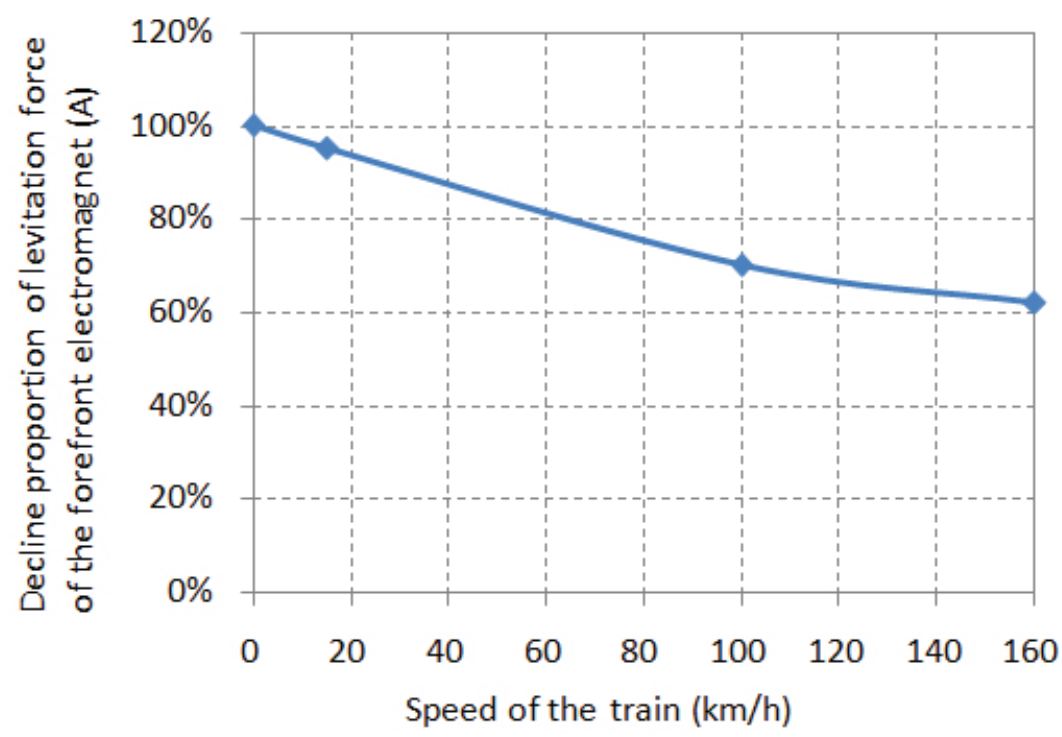

Fig. 5. Relationship between speed and levitation force of forefront electromagnet

\section{FIRST SOLUTION}

To solve the problem of current increasing at the forefront electromagnet caused by the decreasing of levitation force, the most straightforward method is to reduce the load on the forefront electromagnet. The train body and passengers' weight are transmitted to the bogies via the air springs and then to the levitation electromagnets. The pressure of the air springs is controlled in groups, and the pressure of the air springs in one group is the same. Therefore, the area of the air springs above the forefront electromagnets can be reduced to cut down the load on the electromagnets.

As is shown in Fig. 6, every carriage has 20 air springs, divided into 4 groups for control. The pressure in one group is the same. For instance, as for the first group, if we adopt "smaller" air springs above the electromagnets, the load can be divided among the electromagnets that correspond to the other four air springs, and the second group is similar.

In the version 1.0 maglev trains, we conducted comparative tests using "standard" air springs and "smaller" air springs, respectively. The experiments' data is shown in Table 1. It can be seen that with a smaller air springs, the load is reduced by an average of about $19 \%$. 


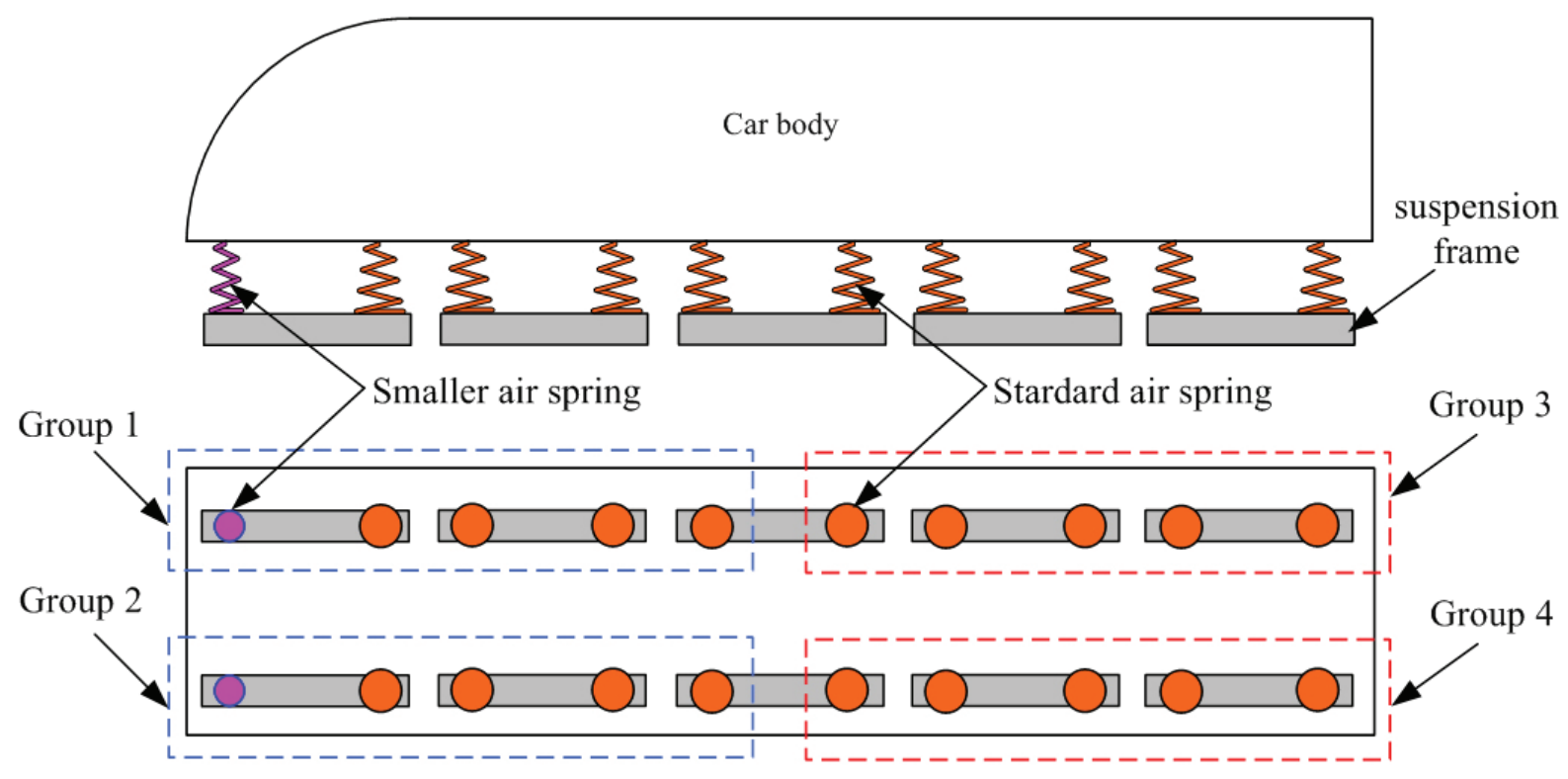

Fig. 6. Arrangement of air springs on the maglev train

Table 1. Load comparative between standard and smaller air springs

\begin{tabular}{|l|c|c|c|c|}
\hline Pressure of air spring (MPa) & 0.4 & 0.5 & 0.6 & 0.7 \\
\hline Load of standard air spring $(\mathrm{kN})$ & 8.5 & 10.7 & 13.0 & 15.3 \\
\hline Load of smaller air spring $(\mathrm{kN})$ & 6.9 & 8.7 & 10.5 & 12.2 \\
\hline Percentage of load decreasing (\%) & 18.8 & 18.7 & 19.2 & 20.3 \\
\hline
\end{tabular}

\section{SECOND SOLUTION}

The second solution is to increase the number of windings of the forefront electromagnets and to lengthen the length of the electromagnet cores synchronously. At present, this solution has been adopted on version 2.0 maglev train, but since the version 2.0 maglev train just went off the assembly line on June $13^{\text {th }}, 2018$ (as shown in Fig. 7), it is still in the commissioning stage, only the data of the train running at low speed is currently available. (As shown in the figure), there is no experimental data when the train is running at a speed of $160 \mathrm{~km} / \mathrm{h}$.

As shown in Fig. 8, the standard levitation electromagnet consists of 4 windings divided into two groups. Two windings in each group are connected in series and controlled by one levitation controller. The extended forefront levitation electromagnet consists of 5 windings. The first group at the front end contains 3 windings controlled by one levitation controller. The second group at the back end contains 2 windings, controlled by another levitation controller. As the number of the first set of windings at the front end increases, so the iron core lengthens, a 

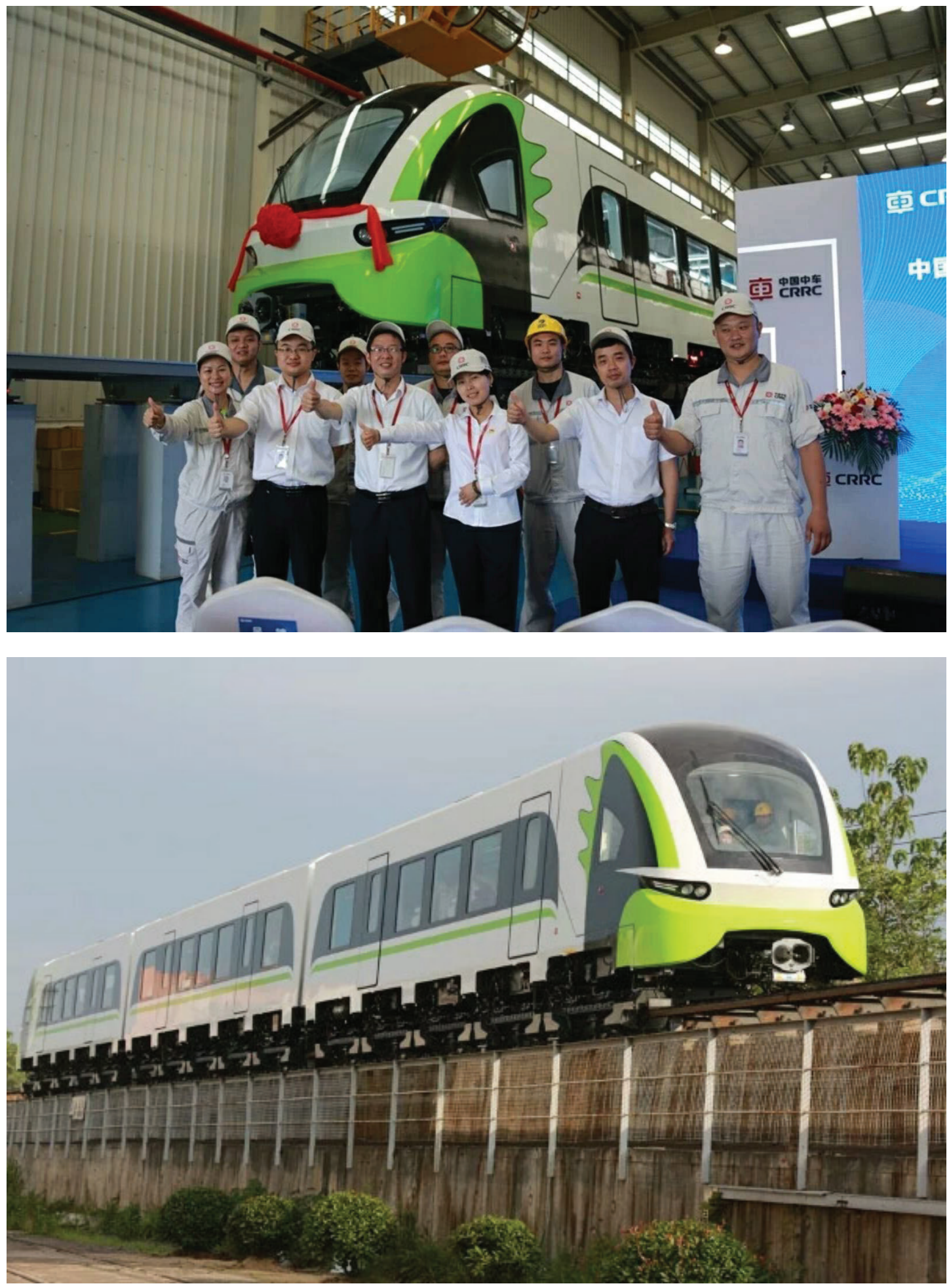

Fig. 7. Version 2.0 commercial maglev train with speed grade of $160 \mathrm{~km} / \mathrm{h}$

larger levitation force can be generated at the side of the three windings at the front end when the same current is passed in, or, under the condition that the load is not changed, the three windings at the front end require smaller current. 

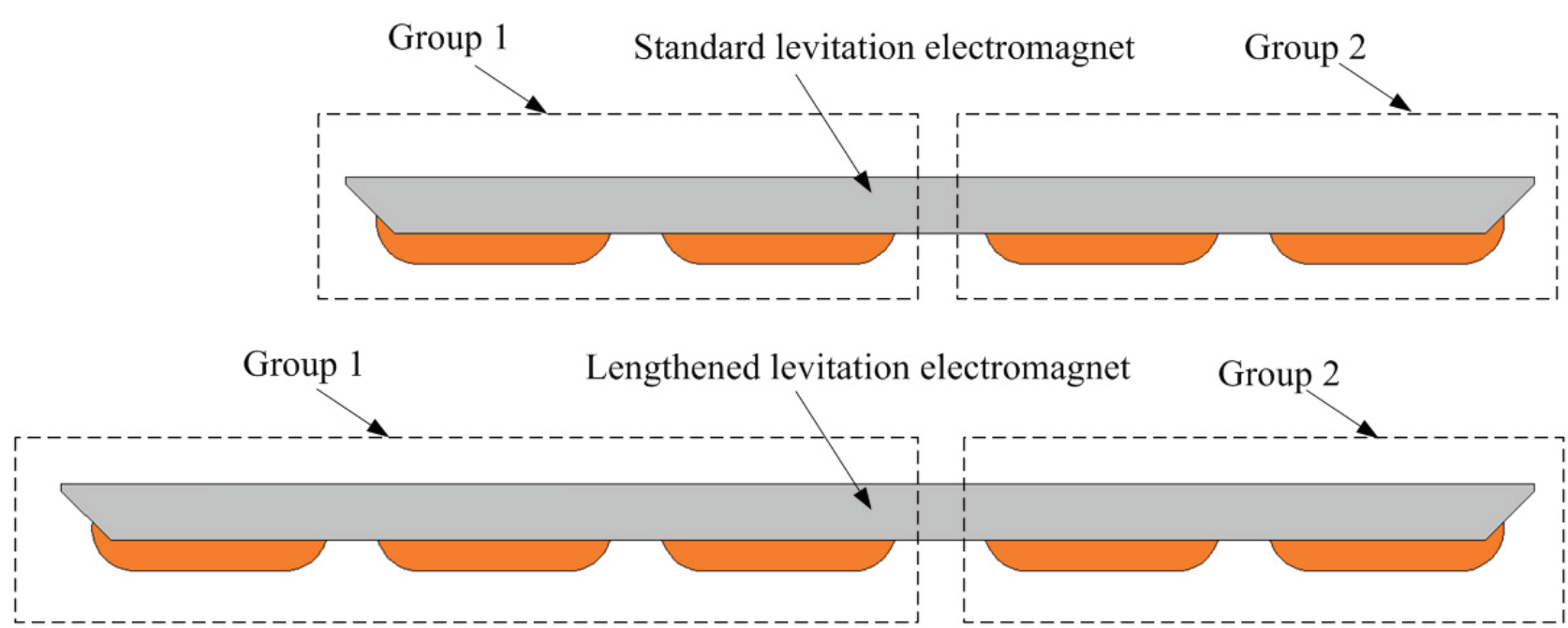

Fig. 8. Comparison of lengthened levitation electromagnets and standard levitation electromagnets

After experiments, the currents of the windings at the front end of the two forefront electromagnets were only $15.12 \mathrm{~A}$ and $16.38 \mathrm{~A}$, while the average currents at other positions were $21.84 \mathrm{~A}$. The average current reduces by $31 \%$.

\section{CONCLUSION}

This paper studied on the problem that the rail eddy current will weaken the levitation force of forefront electromagnets of medium and low speed maglev trains, and proposed two specific solutions and conducted experimental testing. The results show that adopting smaller air springs above the forefront electromagnets or increasing the number of the forefront electromagnets' windings and lengthening the iron core can alleviate the eddy current effects to some extent.

\section{ACKNOWLEDGEMENS}

This paper is supported by the National Key Research and Development Plan (No. 2016YFB1200601).

\section{References}

1. Ohsaki H, Du J. Influence of Eddy Current Induced in Steel Rails on Electromagnetic Force Characteristics of EMS Maglev Systems. Proceedings of the Maglev'2004. 2004;2:960-965. 
2. Zheng L, Li Jie, Li J. Research on the Influence of Eddy Current Induced in Steel Rails on the Levitation Force of a Maglev Train [dissertation]. Changsha (Hunan, P.R.C): National University of Defense Technology; 2010.

Information about the authors:

Ying Yang, Master, Professor Level Senior Engineer;

ORCID: 0000-0002-3125-2126;

E-mail: 2560828867@qq.com

Wenyue Zhang, Ph.D, Senior Engineer;

ORCID: 0000-0002-7005-6283;

E-mail: zhang-wen-yue@126.com

Laisheng Tong, Ph.D, Professor Level Senior Engineer;

ORCID: 0000-0001-6905-5198;

E-mail: alanatlsh@126.com

Qibiao Peng, Bachelor, Professor Level Senior Engineer;

ORCID: 0000-0003-0082-4221;

E-mail: pengqibi@sina.com

Huajun Luo, Master, Professor Level Senior Engineer;

ORCID: 0000-0002-8569-5686;

E-mail: 1484904208@qq.com

Jianguo Suo, Bachelor, Professor Level Senior Engineer;

ORCID: 0000-0003-2669-6926;

E-mail: bajiuwangyue@126.com

\section{To cite this article:}

Yang Y, Zhang W, Tong L, et al. Analysis and Solution of Eddy Current Induced in Rail for Medium and Low Speed Maglev Transportation System. Transportation Systems and Technology. 2018;4(4):129-137. doi: 10.17816/transsyst201844129-137 